\title{
Ground-State Pressure of Quasi-2D Fermi and Bose Gases
}

\author{
Vasiliy Makhalov, Kirill Martiyanov, and Andrey Turlapov ${ }^{*}$ \\ Institute of Applied Physics, Russian Academy of Sciences, ulitsa Ulyanova 46, Nizhniy Novgorod, 603000, Russia \\ (Received 13 May 2013; revised manuscript received 4 November 2013; published 27 January 2014)
}

Using an ultracold gas of atoms, we have realized a quasi-two-dimensional Fermi system with widely tunable $s$-wave interactions nearly in a ground state. Pressure and density are measured. The experiment covers physically different regimes: weakly and strongly attractive Fermi gases and a Bose gas of tightly bound pairs of fermions. In the Fermi regime of weak interactions, the pressure is systematically above a Fermi-liquid-theory prediction, maybe due to mesoscopic effects. In the opposite Bose regime, the pressure agrees with a bosonic mean-field scaling in a range beyond simplest expectations. In the strongly interacting regime, measurements disagree with a purely 2D model. Reported data may serve for sensitive testing of theoretical methods applicable across different quantum physics disciplines.

DOI: 10.1103/PhysRevLett.112.045301

PACS numbers: 67.85.Lm, 03.75.Ss, 67.85.Hj

Two-dimensional many-body quantum systems show interesting physics and are technologically important. In 2D the phenomena of superfluidity and Bose condensation become clearly separated [1]. High-temperature superconductivity is attributed to the 2D structure of the materials [2]. Semiconductor and oxide interfaces containing 2D electron gas are important for modern and prospective electronics [3,4].

The concept of a Bardeen-Cooper-Schrieffer (BCS) to Bose-Einstein-condensate (BEC) crossover $[5,6]$ gives a unified view at some Fermi and Bose systems: By varying interactions, a gas of fermions obeying the BCS or similar model may be smoothly converted into a gas of pointlike bosons, which are pairs of the initial fermions. Such a crossover has been predicted for excitons [7] and quarks [8] and realized in a 3D gas of ultracold fermionic atoms with $s$-wave interactions [9]. Measurements on this system have stimulated development of the many-body quantum theory $[5,6]$, especially for the challenging regime of strong interactions which lies between the BCS and Bose asymptotes.

The 2D BCS-BEC crossover for fermions with $s$-wave interactions is the focus of this Letter. The strongly interacting regime of this crossover may be relevant to hightemperature superconductors: While the superconducting phase of the cuprates has $d$-wave symmetry [2], the $s$-wave symmetry has been detected in the pseudogap phase [10]. Exploring the Bose part of the crossover complements studies of interacting 2D Bose gases [11] by reaching stronger interactions. Studying the fermionic side may add to the understanding of 2D Fermi liquids. Failure of the meanfield description is an example of theoretical challenges in 2D: In 3D the BCS-BEC crossover is qualitatively modeled by a mean field of Cooper pairs (Fig. 5 of Ref. [5]), while in 2D a similar model is qualitatively incorrect, predicting an interaction-independent equation of state at zero temperature [12].
The pure 2D paradigm assumes motion strictly in the $x y$ plane and no $z$ dependence in interactions. In reality, particles experience zero-point oscillations along $z$ and interact via 3D potentials. The term "quasi-2D" generally indicates some departure from the pure 2D approximation while the kinematics remains close to 2D. For example, in most cuprate superconductors, the $2 \mathrm{D}$ picture is altered by interlayer hopping of electrons, but pure 2D models are widespread [2]. An example of a $2 \mathrm{D}$ model insufficiency is ${ }^{3} \mathrm{He}$ on a substrate, where an increase of zero-point oscillations relative to the atom-atom interaction range brings about the formation of a self-bound liquid [13].

Ultracold Fermi atoms $[5,14]$ are well suited for studying the crossover and testing the applicability of purely $2 \mathrm{D}$ models in quasi-2D (Q2D). The atomic system allows an $a b$ initio description because of purity and the knowledge of microscopic and external parameters; e.g., 2D kinematics is achieved by holding atoms in the lowest state of the precisely known potential $m \omega_{z}^{2} z^{2} / 2$ [15], where $m$ is the atom mass. The range of atom-atom interaction is nearly zero, which has two consequences: (i) The $s$-wave collisions are quasi-2D rather than $2 \mathrm{D}$, because at distances $\ll l_{z} \equiv \sqrt{\hbar / 2 m \omega_{z}}$ the two-atom wave function is determined by the 3D scattering length $a$; (ii) the interaction may be mapped onto $s$-wave scattering by a purely $2 \mathrm{D}$ potential [16]; i.e., purely 2D collisions are simulated. There is a controversy, however, in calculating $a_{2}$, the corresponding 2D $s$-wave scattering length, which we resolve below.

We find $a_{2}$ by equating the amplitude of 2D scattering

$$
f_{2 \mathrm{D}}\left(q, a_{2}\right)=-\frac{2 \pi}{\ln \left(q a_{2} e^{\gamma_{E}} / 2 i\right)}
$$

to the scattering amplitude of atoms interacting via 3D contact potential and confined to the lowest state of the potential $m \omega_{z}^{2} z^{2} / 2[16]$ : 


$$
f_{\mathrm{Q} 2 \mathrm{D}}\left(q, a, l_{z}\right)=\frac{2 \pi}{\sqrt{\pi} l_{z} / a+w\left(q^{2} l_{z}^{2}\right) / 2} .
$$

Here $\gamma_{E} \simeq 0.577$ is Euler's constant, $w(\xi)$ is defined in Refs. [16,17], and $\hbar q=\sqrt{2 \mu \mathrm{m}}$ is the relative momentum expressed via the chemical potential $\mu$.

In the alternative approach, similar to Refs. [18,19] and not adopted here, $a_{2}$ is found from binding energy $E_{3 \mathrm{Dbound}}$ of the 3D dimer molecule in the potential $(2 m) \omega_{z}^{2} z^{2} / 2$ [Eq. (17) of Ref. [17]], by equating $E_{3 \mathrm{D} \text { bound }}$ to the binding energy in a 2D potential: $E_{3 \mathrm{D} \text { bound }}=-4 \hbar^{2} / m e^{2 \gamma_{E}} a_{2}^{2}$.

In the limit $\mu \ll \hbar \omega_{z}$, these two approaches give the same $a_{2}$ for small $a<0$. The controversy is for small $a>0$, which is seen by considering the mean field of a uniform 2D gas of atoms. The leading-order term is $-2 \pi \hbar^{2} n_{2} /\left[m \ln \left(a_{2} \sqrt{n_{2}}\right)\right]$ [20]. Plugging in $a_{2}$ derived from $f_{2 \mathrm{D}}=f_{\mathrm{Q} 2 \mathrm{D}}$, one obtains the mean-field value $\sqrt{4 \pi} \hbar^{2} n_{2} a /\left(m l_{z}\right)$ in agreement with Ref. [21], while the bound-state-based method yields a much larger $a_{2}$ overestimating the mean field. This motivates the choice of the amplitude-based approach.

While two-body atom-atom collisions are exactly mapped onto purely 2D interactions, the effect of many-body interactions on dimensionality is unclear. Potentially, strong many-body interactions may alter 2D kinematics by populating excited states of motion along $z$, making the system quasi-2D.

The state of tunable atomic Fermi gases with predominantly $2 \mathrm{D}$ kinematics has been studied by means of radiofrequency spectroscopy $[18,19,22,23]$, measurement of cloud size [24], and observing collective modes [25]. Experiments $[18,22]$ have shown that reduced dimensionality makes pairing more favorable. In Ref. [18], pairbreaking energy in a strongly interacting Fermi system is in agreement with the mean field of Cooper pairs. Alternatively, in Ref. [23], the excitations are inconsistent with the mean-field interpretation and the system is described as a gas of noninteracting polarons. A quantitative interpretation of these and other finite-temperature studies in the Bose and strongly interacting regimes is complicated, because in 2D quantitative thermometry has been available only for weakly interacting Fermi gas $[15,18]$. Observation of many-body effects by means of rf spectroscopy puts stringent requirements on experimental precision, because many-body physics is masked by one- and two-body effects.

In this Letter, we report on the controllable realization and study of the quasi-2D BCS-BEC crossover. The thermometry limitations are circumvented by preparing the system nearly in the ground state. The 2D pressure per spin state $P_{2}$ and the respective numerical planar density $n_{2}$ are measured. Local thermodynamic quantities have been measured in the 3D BCS-BEC crossover [26,27]. In 2D, unlike in 3D, such quantities are sensitive to beyondmean-field effects even at the qualitative level. In particular, as the system becomes more bosonic, the pressure should drop, contrary to the mean-field expectations [12].

The apparatus and gas preparation are generally described in Refs. [15,28] with relevant details elaborated in Ref. [17]. Lithium-6 atoms are equally populating the two lowestenergy spin states $|1\rangle$ and $|2\rangle$. The $s$-wave interactions are controlled by external magnetic field $B$, by using a broad Fano-Feshbach resonance, which in 3D lies at $B=832 G$ [29]. The pancake-shaped trapping potential $V$ is nearly harmonic: $V(\vec{\rho}, z) \simeq\left(\omega_{x}^{2} x^{2}+\omega_{y}^{2} y^{2}+\omega_{z}^{2} z^{2}\right) m / 2$, and tight along $z: \omega_{z} / \omega_{\perp}=52.2 \gg 1$, where $\omega_{\perp} \equiv \sqrt{\omega_{x} \omega_{y}}$ and $\omega_{y} / \omega_{x}=1.50$. A series of such nearly identical potentials is formed by antinodes of a standing wave; 100-200 adjacent traps are loaded. The longitudinal frequency is chosen in the range $\omega_{z} / 2 \pi=2.28-13.7 \mathrm{kHz}$ corresponding to the lattice depth $V_{0}=(1.9-11.6) \hbar \omega_{z}$. The number of atoms per spin state $N$ varied between 180 and 1040. This gives the noninteracting-gas Fermi energy $E_{F}=\hbar \omega_{\perp} \sqrt{2 N}=$ $(0.36-0.87) \hbar \omega_{z}$.

The pressure and density are measured in the locally homogeneous part of the cloud, near the center $\rho=0$, by analyzing the linear density profiles $n_{1}(x)$ as the one in Fig. 1(a). These profiles are obtained by imaging [17] the flat clouds from a side, along the $y$ direction, which integrates the density giving the linear distribution $n_{1}(x)=\int n_{2}(\vec{\rho}) d y$. We average $n_{1}(x)$ over 15-30 nearly identical clouds.

The local pressure is obtained from the force balance equation $\nabla_{\perp} P_{2}(\vec{\rho})=-n_{2}(\vec{\rho}) \nabla_{\perp} V(\vec{\rho}, z)$. Integrating, one finds the central pressure $P_{2}=m \omega_{\perp}^{2} N\left(1-m \omega_{x}^{2}\left\langle x^{2}\right\rangle /\right.$ $\left.V_{0}\right) / 2 \pi$, where the transverse potential is expanded up to the quartic term and $\left\langle x^{2}\right\rangle=(1 / N) \int x^{2} n_{1}(x) d x$. The planar density profile $n_{2}(\vec{\rho})$ is found by performing, first, noise filtering of $n_{1}(x)$ and then the inverse Abel transform adjusted for elliptic clouds [17]. In Fig. 1(b), one may see the planar density distribution in stretched coordinates
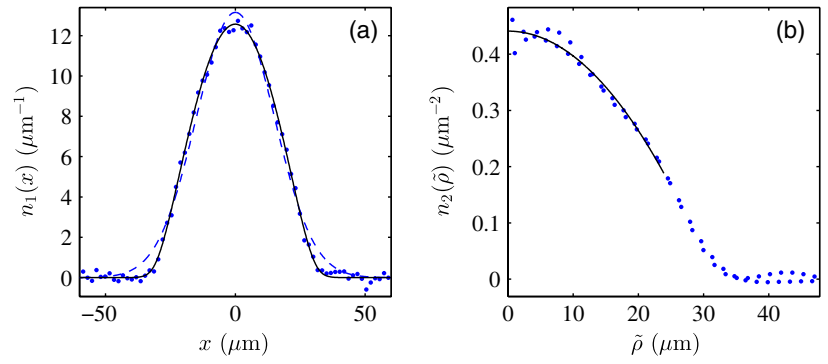

FIG. 1 (color online). (a) Linear density profile $n_{1}(x)$, no noise filtering applied. Dots are the data for $a_{2} \sqrt{n_{2}}=0.89$ $\left(B=850 \mathrm{G}, N=500 \pm 10, l_{z}=-0.29 a\right)$. Solid and dashed curves are the fits by Thomas-Fermi and Gaussian distribution, respectively. The Gaussian fit is off, which proves deep degeneracy. (b) Surface density $n_{2}(\tilde{\rho})$ derived from noise-filtered $n_{1}(x)$. Dots are the data. The curve is the fit of the parabola $n_{2}(\tilde{\rho})=n_{2}-\tilde{\rho}^{2} n_{2}^{\prime \prime} / 2$ to the data, which yields the central density $n_{2} \equiv n_{2}(\tilde{\rho}=0)$. 
$\overrightarrow{\tilde{\rho}}=\left(x, y \omega_{y} / \omega_{x}\right)$, in which the clouds are cylindrically symmetric. For Eq. (2), the value of $\mu$ is needed. The scale-invariant assumption $\mu \propto n_{2}$ together with $d P_{2}=$ $n_{2} d \mu$ gives the estimate $\mu=E_{F} \sqrt{P_{2} / P_{2} \text { ideal }}$, where $P_{2 \text { ideal }}=\pi n_{2}^{2} \hbar^{2} / m$ is the pressure of an ideal Fermi gas of the same density $n_{2}$. This estimate is of sufficient precision, because departure from $\mu \propto n_{2}$ is small [17] and the function $w(\xi)$ is slow.

The dependence of the normalized pressure on the interaction parameter $a_{2} \sqrt{n_{2}}$ is shown in Fig. 2, which is the main result of the Letter. The error bars include statistical and systematic errors [17]. All known systematic effects, except finite temperature, are corrected for as explained in Ref. [17].

Interaction parameter $a_{2} \sqrt{n_{2}}$ is the ratio of the scattering spatial scale to the interparticle distance. Three regimes may be distinguished: (i) For $a_{2} \sqrt{n_{2}} \gg 1$, the system is fermionic because the Fermi pressure dominates over the interactions; (ii) at $a_{2} \sqrt{n_{2}} \sim 1$, the interaction energy is comparable to the Fermi energy, and the system is strongly interacting; (iii) for $a_{2} \sqrt{n_{2}} \ll 1$, the pairing energy is even larger, and the fermions are bound into compact bosonic pairs; the interaction of pairs is small in comparison to the Fermi energy; therefore, the system is a weakly repulsive Bose gas of molecules. The borders between regimes may be taken approximately at $a_{2} \sqrt{n_{2}}=1 / 4$ and 4

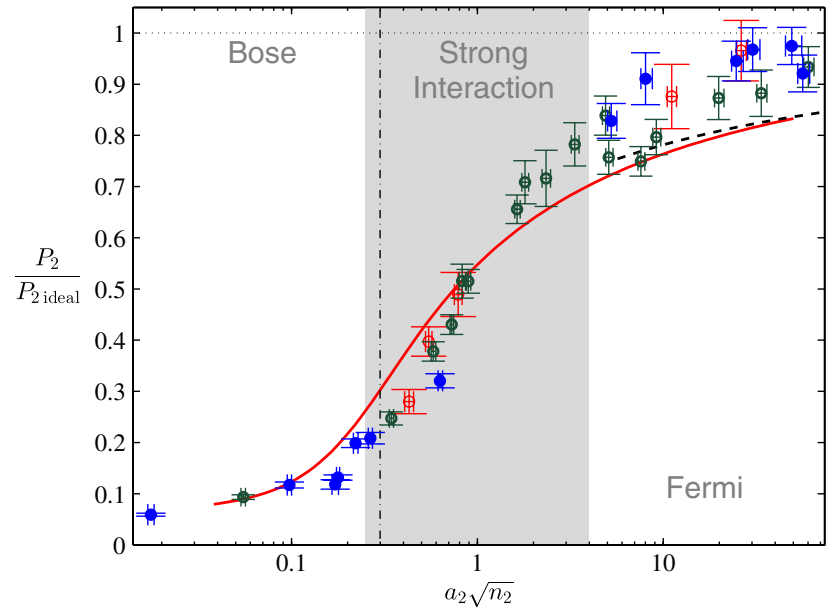

FIG. 2 (color online). Normalized local pressure vs interaction parameter. Data are coded in color by the atom number $N$ : smallest $N=220-350\left[E_{F}=(0.40-0.51) \hbar \omega_{z}\right]$ are shown by empty red circles, intermediate $N=410-560\left[E_{F}=(0.54-0.64) \hbar \omega_{z}\right]$ by green circles with a thicker border, and largest $N=$ 640-910 $\left[E_{F}=(0.69-0.82) \hbar \omega_{z}\right]$ by blue solid dots. Solid red curve: Smooth approximation of the pure 2D Monte Carlo simulation [12]. Dashed curve: Model [30] of a homogeneous 2D Fermi liquid at $T=0$. Dotted line: Mean-field model based on the BCS state [31]. The vertical dash-dotted line separates data into two regions: $a_{2}<l_{z}$ on the left and $a_{2}>l_{z}$ on the right. The data in table form are in Ref. [17].
(Fig. 2). In further discussion, the borders are attached to the data points, which are closest to these two values.

In the Fermi region $a_{2} \sqrt{n_{2}} \geq 4.9$, the ratio $P_{2} / P_{2 \text { ideal }}$ is approaching unity as expected (Fig. 2). Temperatures are evenly distributed in the range $T=(0.02-0.15) E_{F}$, with a confidence interval $\simeq \pm 0.03 E_{F}$ in each measurement. The temperature in the units of the local Fermi energy $\varepsilon_{F}=$ $2 \pi n_{2} \hbar^{2} / m$ is also known, because $\varepsilon_{F} \simeq E_{F}$ in this regime. At $T=0.08 \varepsilon_{F}$ for a weakly attractive Fermi gas, the gap should be closed for $a_{2} \sqrt{n_{2}} \geq 0.54$ [32]. Thus, in the Fermi region the system is likely a Fermi liquid.

For $a_{2} \sqrt{n_{2}} \geq 4.9$, the pressure on average is $10 \%$ above the prediction for a homogeneous 2D Fermi liquid [30], however. Neither an unaccounted population of excited states for the motion along $z$ nor a pairing gap would explain higher pressure, because these effects may only reduce $P_{2} / P_{2 \text { ideal }}$. Finite temperature cannot be the reason either: For an ideal uniform Fermi gas, the pressure rises by $2 \%$ when $T / \varepsilon_{F}$ increases from 0 to 0.08 , which gives an estimate for the effect of temperature on the Fermi-liquid pressure. The observed high pressure could be attributed to the mesoscopic character of the system at large $a_{2}$ values. Whenever $a_{2}$ is larger than the rms cloud size $\sqrt{\left\langle\rho^{2}\right\rangle}$, the interaction is effectively suppressed, which tunes the gas closer to noninteracting. In the Fermi regime, at the cloud center $a_{2} / \sqrt{\left\langle\rho^{2}\right\rangle} \simeq a_{2} \sqrt{n_{2}} \sqrt{1.5 \pi / N}$. For $N=500$, $a_{2} \sqrt{n_{2}}=10$ is the crossover point between the locally homogeneous and mesoscopic regimes. According to this criterion, in Ref. [19] the system is mesoscopic for the weakest interactions, but one-particle excitations are found to agree with a model of a finite-temperature locally homogeneous Fermi liquid, which contradicts our pressure measurements.

As the system becomes more bosonic, the ratio $P_{2} / P_{2 \text { ideal }}$ decreases following qualitative expectations (Fig. 2). In the Bose regime $a_{2} \sqrt{n_{2}} \ll 1$, we find the scaling $P_{2} / P_{2 \text { ideal }} \propto$ $a / l_{z}$ as seen in Fig. 3, where additional data are also shown in the deep Bose regime $a_{2} \sqrt{n_{2}}<0.01$. To understand this scaling, one may note that the leading term in the pressure of Bose molecules should be the same as for pointlike bosons: $P_{2 \text { Bose }}=-P_{2 \text { ideal }} / \ln \left(a_{2 \mathrm{~mol}}^{2} n_{2}\right)$ [20]. Here $a_{2 \mathrm{~mol}}$ is the 2D scattering length for molecule-molecule collisions, which may be related to the respective 3D scattering length $a_{\text {mol }}=0.6 a$ [33] by equating the scattering amplitudes $f_{2 \mathrm{D}}\left(2 q, a_{2 \mathrm{~mol}}\right)=f_{\mathrm{Q} 2 \mathrm{D}}\left(2 q, a_{\mathrm{mol}}, l_{z} / \sqrt{2}\right)$. The pressure calculated for each datum as $P_{2}=P_{2 \text { Bose }} / 2$ is shown in Fig. 3 as the broken solid line. To further simplify $P_{2 \text { Bose }}$, one may take the low-energy limit $2 \mu \ll \hbar \omega_{z}$, yielding $a_{2 \mathrm{~mol}} \simeq$ $2.09 l_{z} \exp \left(-\sqrt{(\pi / 2)}\left(l_{z} / a_{\text {mol }}\right)\right)$, and the limit of unmodified 3D interactions $a_{\text {mol }} \ll l_{z}$, which all together give $P_{2 \text { Bose }} \simeq 2 P_{2 \text { ideal }}\left(a_{\text {mol }} / l_{z} \sqrt{8 \pi}\right)$ shown by the dashed line in Fig. 3. This expression gives the scaling $P_{2} / P_{2 \text { ideal }} \propto a / l_{z}$, though in the data the scaling coefficient is $19 \%$ higher. Unexpectedly, the data agree with this 


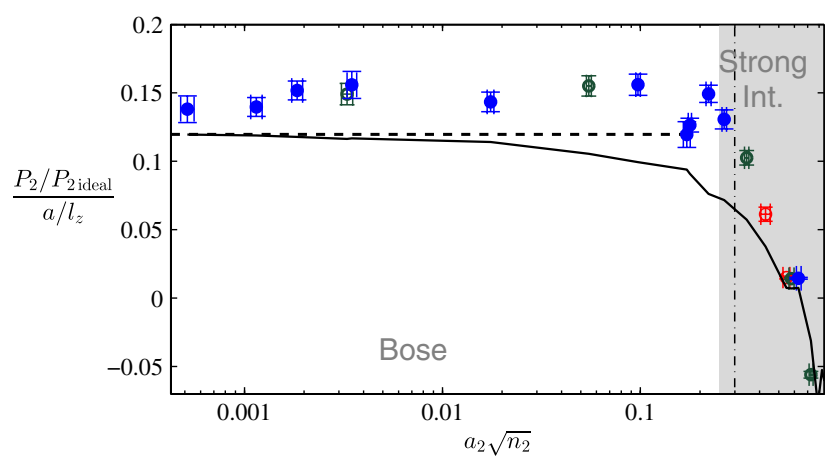

FIG. 3 (color online). Demonstration of linear scaling $P_{2} / P_{2 \text { ideal }} \propto a / l_{z}$ in the Bose regime. Markers: The data $\left(P_{2} / P_{2 \text { ideal }}\right) /\left(a / l_{z}\right)$ vs the interaction parameter. Color coding and the vertical dash-dotted line are the same as in Fig. 2. Dashed horizontal line: Model for pointlike molecular bosons with 3D interactions, $P_{2}=P_{2 \text { ideal }}\left(0.6 a / l_{z} \sqrt{8 \pi}\right)$. The solid line connects points calculated via $P_{2}=P_{2 \text { Bose }} / 2$.

bosonic mean-field scaling beyond $a_{\text {mol }} \ll l_{z}$, up to $a_{\mathrm{mol}} / l_{z}=0.96\left(a_{2} \sqrt{n_{2}}=0.27\right)$ or, in the language of the bosonic coupling parameter $g=-2 \pi / \ln \left(a_{2 \operatorname{mol}} \sqrt{n_{2}}\right)$ [11], up to $g=2.9$. The agreement extends into the border with the strongly interacting region, where the picture of pointlike bosons is questionable.

In the Bose regime $a_{2} \sqrt{n_{2}} \leq 0.22$, to assure the closeness to the ground state, we measure the temperature fitting the $n_{1}(x)$ data by bimodal distribution, which is the sum of a Gaussian and zero-temperature Thomas-Fermi distribution $\left(8 N_{0} / 3 \pi R_{\mathrm{TF}}\right)\left(1-x^{2} / R_{\mathrm{TF}}^{2}\right)^{3 / 2}$, where $R_{\mathrm{TF}}$ and $N_{0}$ are varied. The temperature is inferred from the ideal-Bose-gas relation $N_{0} / N=1-\left(T / T_{\mathrm{cr}}\right)^{2}$, where $T_{\text {cr }}=E_{F} \sqrt{3} / \pi$. This procedure may overestimate the temperature [34]. Fitting consistently yields $T<0.5 T_{\mathrm{cr}}$. To find $T$ in the local units of $\varepsilon_{F}$, we note that $E_{F} / \varepsilon_{F}=\sqrt{P_{2} / P_{2 \text { ideal }}}$. Combining this with the asymptote $P_{2} / P_{2 \text { ideal }}=0.14 a / l_{z}$ seen in Fig. 3, we obtain the upper bound $T / \varepsilon_{F}<0.1 \sqrt{a / l_{z}}$. For the absolute majority of the bosonic data, the temperature is below the BerezinskiiKosterlitz-Thouless transition calculated for weakly interacting bosons [35]. In a few cases, which we checked in the deep Bose regime, BECs are observed: After release of the gas from the lattice and free expansion for a few milliseconds, straight interference fringes are clearly visible. Indeed, finite Bose systems are predicted to condense $[20,36]$. The interference, however, has not been studied systematically for all $a_{2} \sqrt{n_{2}}$. Since $T / \varepsilon_{F}$ is low, the actual phase state, whether Berezinskii-Kosterlitz-Thouless or BEC, may not affect the pressure significantly: The pressure is continuous over these transitions and, therefore, close to the ground-state value.

In the strongly interacting regime $0.27 \leq a_{2} \sqrt{n_{2}} \leq 3.3$, quantitative thermometry is presently unavailable. For demonstrating closeness to the ground state, we act along the lines of empirical thermometry [37]. The density profiles resemble those of the ideal Fermi gas: For the lowest temperatures the edges are sharp, while as $T$ increases the shape transforms into a Gaussian. By fitting the ideal Fermi gas profile [15] to the data $n_{1}(x)$, as in Fig. 1(a), we find the empirical temperature parameter evenly distributed in the range $\left(T / E_{F}\right)_{\text {fit }}=0.02-0.16$, indicating deep degeneracy.

For the regime of strong interactions $a_{2} \sqrt{n_{2}} \sim 1$, in Fig. 2, one may compare the pressure to the prediction of zero-temperature Monte Carlo model [12] for a uniform gas with 2D atom-atom interactions. Some data are lying on the Monte Carlo curve. When the overall trend is considered, one may see that the slope of the data is steeper (also in Fig. 7 of Ref. [17]). Contrary to our findings, measurements of the cloud size [24] in the strongly interacting regime are reported to quantitatively agree with the Monte Carlo results [12]. If the data of Fig. 2 are plotted by using the definition of $a_{2}$ adopted in Ref. [24], the pressure lies systematically below the Monte Carlo curve in most of the strong-interaction region (Fig. 8 of Ref. [17]). In Ref. [24], the system might be significantly away from the ground state: Unlike here in Fig. 1(a), images of the trapped cloud in Ref. [24] do not show the sharp edge.

In the strongly interacting and Fermi regimes of our experiment, the chemical potential $\mu$ is less than but comparable to $\hbar \omega_{z}$. To see whether the closeness of $\mu$ to the excited state matters, we have done measurements with different atom numbers as indicated by color coding in Figs. 2 and 3. Within current precision, there is no dependence on $N$ or $E_{F} / \hbar \omega_{z}$. In addition, the data of Fig. 2 are fitted by a smooth curve $p_{2 \text { fit }}\left(a_{2} \sqrt{n_{2}}\right)$ in the range $0.055 \leq a_{2} \sqrt{n_{2}} \leq 60$. In Fig. 4, we show the ratio of the measured values to this fit: There are no systematic shifts between the points with low and high $\mu / \hbar \omega_{z}$. Also, we rule out two possible sources for the excited state population: (i) There are no thermal excitations [15]; (ii) for twofermion collisions, scattering into the upper states of motion along $z$ is prohibited by energy and parity conservation, because the collision kinetic energy $2 \mu$ is $<2 \hbar \omega_{z}$. By ruling out the simplest reasons of the excited state

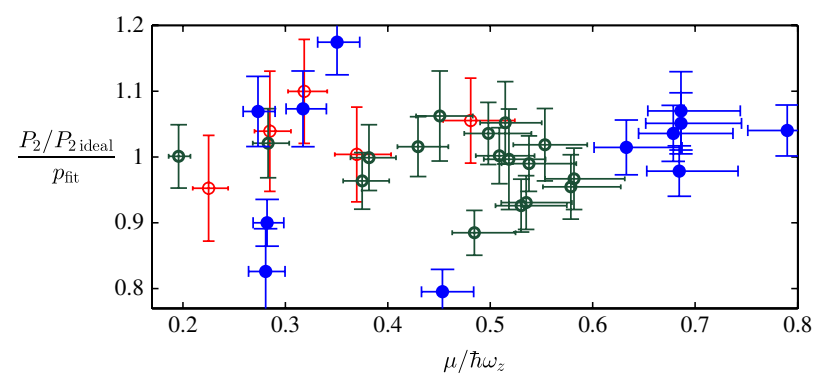

FIG. 4 (color online). Ratio of the measured normalized pressure to the respective values of a smooth fitting function [17] vs the normalized chemical potential. Color coding is the same as in Fig. 2. 
population, we do not exclude such a population completely, because it may be induced by strong interactions. Such quasi-2D effects are a potential reason for deviation from the pure 2D Monte Carlo model [12].

In conclusion, a widely tunable quasi-2D Fermi system nearly in the ground state has been realized experimentally. The pressure measurements may be used for sensitive testing of many-body theories including the question of applicability of purely 2D models in strongly interacting systems.

We are thankful to D. S. Petrov for discussions. We acknowledge the financial support by the programs of the Presidium of Russian Academy of Sciences "Quantum mesoscopic and disordered structures" and "Nonlinear dynamics" and Russian Foundation for Basic Research (Grants No. 11-02-01324-a, No. 11-02-12282ofi-m-2011, and No. 12-02-31804 mol_a).

*turlapov@appl.sci-nnov.ru

[1] V. L. Berezinskii, Sov. Phys. JETP 34, 610 (1972).

[2] V. M. Loktev, R. M. Quick, and S. G. Sharapov, Phys. Rep. 349, 1 (2001).

[3] T. Ando, A. B. Fowler, and F. Stern, Rev. Mod. Phys. 54, 437 (1982).

[4] J. Mannhart, D. H. A. Blank, H. Y. Hwang, A. J. Millis, and J.-M. Triscone, MRS Bull. 33, 1027 (2008).

[5] S. Giorgini, L. P. Pitaevskii, and S. Stringari, Rev. Mod. Phys. 80, 1215 (2008).

[6] M. Randeria and E. Taylor, arXiv:1306.5785.

[7] L. V. Keldysh and A. N. Kozlov, Sov. Phys. JETP 27, 521 (1968).

[8] B. O. Kerbikov, Phys. At. Nucl. 65, 1918 (2002).

[9] M. Bartenstein, A. Altmeyer, S. Riedl, S. Jochim, C. Chin, J. H. Denschlag, and R. Grimm, Phys. Rev. Lett. 92, 120401 (2004).

[10] S. Sakai, S. Blanc, M. Civelli, Y. Gallais, M. Cazayous, M.-A. Méasson, J.S. Wen, Z. J. Xu, G. D. Gu, G. Sangiovanni, Y. Motome, K. Held, A. Sacuto, A. Georges, and M. Imada, Phys. Rev. Lett. 111, 107001 (2013).

[11] L.-C. Ha, C.-L. Hung, X. Zhang, U. Eismann, S.-K. Tung, and C. Chin, Phys. Rev. Lett. 110, 145302 (2013).

[12] G. Bertaina and S. Giorgini, Phys. Rev. Lett. 106, 110403 (2011).

[13] M. Ruggeri, S. Moroni, and M. Boninsegni, Phys. Rev. Lett. 111, 045303 (2013).

[14] I. Bloch, J. Dalibard, and W. Zwerger, Rev. Mod. Phys. 80, 885 (2008).
[15] K. Martiyanov, V. Makhalov, and A. Turlapov, Phys. Rev. Lett. 105, 030404 (2010).

[16] D. S. Petrov and G. V. Shlyapnikov, Phys. Rev. A 64, 012706 (2001).

[17] See Supplemental Material at http://link.aps.org/ supplemental/10.1103/PhysRevLett.112.045301 for gas preparation; details on finding the planar density profile $n_{2}(\vec{\rho})$; error analysis; $w(\xi)$ definition; fit to the data; data representation for alternative definitions of $a_{2}$; and data and experimental parameters in table form.

[18] M. Feld, B. Fröhlich, E. Vogt, M. Koschorreck, and M. Köhl, Nature (London) 480, 75 (2011).

[19] B. Fröhlich, M. Feld, E. Vogt, M. Koschorreck, M. Köhl, C. Berthod, and T. Giamarchi, Phys. Rev. Lett. 109, 130403 (2012).

[20] M. Schick, Phys. Rev. A 3, 1067 (1971).

[21] L. Pricoupenko, Phys. Rev. A 70, 013601 (2004).

[22] A. T. Sommer, L. W. Cheuk, M. J. H. Ku, W. S. Bakr, and M. W. Zwierlein, Phys. Rev. Lett. 108, 045302 (2012).

[23] Y. Zhang, W. Ong, I. Arakelyan, and J. E. Thomas, Phys. Rev. Lett. 108, 235302 (2012).

[24] A. A. Orel, P. Dyke, M. Delehaye, C. J. Vale, and H. Hu, New J. Phys. 13, 113032 (2011).

[25] E. Vogt, M. Feld, B. Fröhlich, D. Pertot, M. Koschorreck, and M. Köhl, Phys. Rev. Lett. 108, 070404 (2012).

[26] J. Joseph, B. Clancy, L. Luo, J. Kinast, A. Turlapov, and J. E. Thomas, Phys. Rev. Lett. 98, 170401 (2007).

[27] N. Navon, S. Nascimbene, F. Chevy, and C. Salomon, Science 328, 729 (2010).

[28] K. A. Martiyanov, V. B. Makhalov, and A. V. Turlapov, JETP Lett. 91, 369 (2010).

[29] G. Zürn, T. Lompe, A. N. Wenz, S. Jochim, P. S. Julienne, and J. M. Hutson, Phys. Rev. Lett. 110, 135301 (2013).

[30] P. Bloom, Phys. Rev. B 12, 125 (1975).

[31] M. Randeria, J.-M. Duan, and L.-Y. Shieh, Phys. Rev. Lett. 62, 981 (1989).

[32] D. S. Petrov, M. A. Baranov, and G. V. Shlyapnikov, Phys. Rev. A 67, 031601 (2003).

[33] D. S. Petrov, C. Salomon, and G. V. Shlyapnikov, Phys. Rev. Lett. 93, 090404 (2004).

[34] Z. Hadzibabic, P. Krüger, M. Cheneau, S. R. Rath, and J. Dalibard, New J. Phys. 10, 045006 (2008).

[35] N. Prokof'ev, O. Ruebenacker, and B. Svistunov, Phys. Rev. Lett. 87, 270402 (2001).

[36] Y. Kagan, B. V. Svistunov, and G. V. Shlyapnikov, Sov. Phys. JETP 66, 314 (1987).

[37] J. Kinast, A. Turlapov, J. E. Thomas, Q. Chen, J. Stajic, and K. Levin, Science 307, 1296 (2005). 